\title{
STOCHASTIC PROCESSES ASSOCIATED WITH THERMAL QUASI-FREE STATES
}

\author{
ROMAN GIELERAK, LECH JAKÓBCZYK and ROBERT OLKIEWICZ \\ Institute of Theoretical Physics, University of Wroctaw \\ pl. M. Borna 9, 50-204 Wroctaw, Poland \\ E-mail: gielerak@ift.uni.wroc.pl
}

1. Introduction. In the present note, we introduce a general conceptual framework for so called stochastically positive structures on Weyl algebras, which are described by some stochastic processes. As a starting point for further development, we analyse in detail the case of quasi-free states. We completely characterize stochastically positive quasi-free KMS states and study the properties of corresponding periodic stochastic processes. In particular, we show that in that case, all relevant information about KMS structure is contained in the commutative sector given by a thermal process since the modular structure given by a stochastic process is unitarily equivalent with the canonical modular structure associated with KMS state. Let us emphasize that this gives new possibilities for the description of KMS structures in the case when interaction is present. Having described quasi-free systems in terms of stochastic processes, one may perturb them with multiplicative-like functionals, thereby creating some new non-Gaussian thermal process. Furthermore, given such a process, we can reproduce its KMS structure. Some results in this direction were obtained in [3] for gentle perturbations of the free Bose gas in the noncritical region of densities and in [4] for the critical case. We present here an application of our results to the case of the scalar quantum field theory on a globally hyperbolic stationary space-time, and we give a general result on the existence of a Markov thermal process, which determines the whole modular structure of the theory. In the case of a static space-time, we obtain much more explicit description of the arising process and we are able to discuss its continuity properties.

2. Analytic continuation of quasi-free KMS states. Let $\mathcal{D}$ be a complex Hilbert space with a scalar product $\langle\cdot, \cdot\rangle$ and let $\sigma(f, g)=\operatorname{Im}\langle f, g\rangle$. Denote by $W(\mathcal{D}, \sigma)$ the Weyl

1991 Mathematics Subject Classification: Primary 82B10; Secondary 60G20.

Research supported by KBN grant 2PO3B 12211

The paper is in final form and no version of it will be published elsewhere. 
algebra over $(\mathcal{D}, \sigma)([10])$ generated by elements $W_{f}, f \in \mathcal{D}$ satisfying

$$
W_{f} W_{g}=e^{-\sigma(f, g) / 2} W_{f+g}
$$

If $T_{t}$ is a group of unitary operators on $\mathcal{D}$ with a generator $\mathbf{h}$ and if $B(f, g)$ is a positive, sesquilinear form on $\mathcal{D}$ which is $T_{t}$ invariant and satisfies

$$
|\sigma(f, g)|^{2} \leq B(f, f) B(g, g)
$$

then $\omega$ defined by

$$
\omega\left(W_{f}\right)=e^{-\frac{1}{4} B(f, f)}, \quad W_{f} \in W(\mathcal{D}, \sigma)
$$

and extended by linearity and continuity to the whole $W(\mathcal{D}, \sigma)$ is a gauge invariant quasi-free state on $W(\mathcal{D}, \sigma)$. In that case the two point correlation functions

$$
H(f, g ; t)=\omega\left(W_{f} W_{T_{t} g}\right)
$$

have the form

$$
H(f, g ; t)=e^{-\frac{1}{4}(B(f, f)+B(g, g))-\frac{1}{2} F(f, g ; t)}
$$

where

$$
F(f, g ; t)=\operatorname{Re} B\left(f, T_{t} g\right)+i \sigma\left(f, T_{t} g\right)
$$

If $\omega$ is a KMS state with respect to the evolution on $W(\mathcal{D}, \sigma)$ generated by $T_{t}$ at the inverse temperature $\beta([1])$, then for every $f, g \in \mathcal{D}$ there exists a function $\Phi(f, g ; z)$ analytic in the strip $0<\operatorname{Im} z<\beta$, such that

$$
\Phi(f, g ; t+i 0)=F(f, g ; t), \quad \text { and } \quad \Phi(f, g ; t+i \beta)=F(g, f ;-t)
$$

Starting with the functions $\Phi(f, g ; z)$ we can define Euclidean-time correlation functions $G(f, g ; s)$ by the formula

$$
G(f, g ; s)=e^{-\frac{1}{4}(B(f, f)+B(g, g))-\frac{1}{2} S(f, g ; s)}
$$

where

$$
S(f, g ; s)=\Phi(f, g ; i s), \quad s \in[0, \beta]
$$

and

$$
G\left(f_{1}, \ldots, f_{n} ; s_{1}, \ldots, s_{n}\right)=\prod_{k=1}^{n} e^{-\frac{1}{4} B\left(f_{k}, f_{k}\right)} \prod_{\left(j_{k}, l_{k}\right)} e^{-\frac{1}{2} S\left(f_{j_{k}}, f_{l_{k}} ; s_{l_{k}}-s_{j_{k}}\right)}
$$

where the second product is taken over all pairs of different indices $\left(j_{k}, l_{k}\right)$ such that $j_{k}, l_{k} \in\{1, \ldots, n\}, s_{j_{k}}<s_{l_{k}}$. Extending (10) by linearity and continuity to arbitrary $w_{1}, \ldots, w_{n} \in W(\mathcal{D}, \sigma)$ we obtain Euclidean-time correlation functions $G\left(w_{1}, \ldots, w_{n}\right.$; $\left.s_{1}, \ldots, s_{n}\right)$.

Proposition 1 ([2]). The functions $S(f, g ; s)$ defined by $(9)$ satisfy:

1. for every $f, g \in \mathcal{D}$ and $s \in[0, \beta]$

$$
S(f, g ; s)=S(g, f ; \beta-s)
$$

2. for every $f \in \mathcal{D}$ the mapping $s \rightarrow S(f, f ; s)$ is OS-positive, i.e. for any sequences $\left\{s_{k}\right\}, s_{k} \in[0, \beta / 2]$ and $\left\{c_{k}\right\}, c_{k} \in \mathbb{C}$

$$
\sum_{k, l} \bar{c}_{k} c_{l} S\left(f, f ; s_{k}+s_{l}\right) \geq 0
$$


More generally, for any terminating sequences $\left\{f_{k}\right\}, f_{k} \in \mathcal{D} ;\left\{s_{k}\right\}, s_{k} \in[0, \beta / 2]$ and $\left\{c_{k}\right\}, c_{k} \in \mathbb{C}$

$$
\sum_{k, l} \bar{c}_{k} c_{l} S\left(f_{k}, f_{l} ; s_{k}+s_{l}\right) \geq 0
$$

3. Stochastic positivity and thermal processes. For a general KMS state $\omega$ on an abstract $\mathrm{C}^{*}$-algebra $\mathcal{A}$, Klein and Landau [7] discussed the problem of construction of a stochastic process corresponding to $\omega$. As they showed, such a process can be constructed using some abelian sub- ${ }^{*}$-algebra $\mathcal{B}$ of $\mathcal{A}$ on which correlation functions at Euclidean times are positive in some special sense. In such case, the process has values in the spectrum of the abelian algebra $\mathcal{B}$. To study the existence of a stochastic process in the case of the Weyl algebra $W(\mathcal{D}, \sigma)$, we consider abelian subalgebras of $W(\mathcal{D}, \sigma)$ defined in terms of so called abelian splitting of the symplectic space $(\mathcal{D}, \sigma)$.

Definition 1. A pair $\left(\mathcal{D}_{+}, \mathcal{D}_{-}\right)$of linear subspaces of the symplectic space $(\mathcal{D}, \sigma)$ is called an abelian splitting if $\mathcal{D}=\mathcal{D}_{+}+\mathcal{D}_{-}$and $\sigma\left(\mathcal{D}_{ \pm}, \mathcal{D}_{ \pm}\right)=0$. For the abelian splitting $\left(\mathcal{D}_{+}, \mathcal{D}_{-}\right)$, let $W_{+}$and $W_{-}$be the abelian subalgebras of $W(\mathcal{D}, \sigma)$ generated by $W_{f}$ with $f \in \mathcal{D}_{+}$and $\mathcal{D}_{-}$respectively.

Now the desired positivity condition is the following:

Definition 2. The set of Euclidean-time correlation functions on the Weyl algebra $W(\mathcal{D}, \sigma)$ with the given abelian splitting is $W_{ \pm}$-stochastically positive if for any positive elements $w_{1}, \ldots, w_{n} \in W_{ \pm}$and $-\beta / 2 \leq s_{1} \leq \cdots \leq s_{n} \leq \beta / 2$

$$
G\left(w_{1}, \ldots, w_{n} ; s_{1}, \ldots, s_{n}\right) \geq 0
$$

Stochastic positivity of quasi-free correlation functions can be naturally formulated in terms of the functions $S(f, g ; s)$. Let $C$ be an abstract complex conjugation on $\mathcal{D}$ i.e. $C$ is antiunitary and $C^{2}=1$. The mapping $C$ defines the abelian splitting $\left(\mathcal{D}_{+}, \mathcal{D}_{-}\right)$of $\mathcal{D}$ :

$$
\mathcal{D}_{+}=\{f \in \mathcal{D}: C f=f\}, \quad \mathcal{D}_{-}=\{f \in \mathcal{D}: C f=-f\}
$$

Proposition 2 ([2]). If the generator $\mathbf{h}$ of $T_{t}$ is $C$-real (i.e. if $C$ leaves the domain $D(\mathbf{h})$ invariant and $C \mathbf{h} f=\mathbf{h} C f$ for every $f \in D(\mathbf{h}))$, then the functions $S(f, g ; s)$ restricted to $\mathcal{D}_{+}$satisfy:

$$
S(f, g ; s)=S(g, f ; s), \quad s \in[0, \beta]
$$

Combining this result with that of Proposition 1 we get

$$
S(f, g ; s)=S(f, g ; \beta-s)
$$

This allows us to extend the function $S(f, g ; s)$ (for the fixed $f, g \in \mathcal{D}_{+}$) to a periodic function of $s$ with the period $\beta$, defined for all $s \in \mathbb{R}$. The extended function will also be denoted by the same symbol.

THEOREM 3. $S(f, g ; s)$ defines an operator-valued covariance function $R_{\beta}(s)$ of a periodic Gaussian OS-positive stochastic process indexed by $\mathcal{D}_{+}$. Thus $R_{\beta}(s)$ is an operatorvalued positive-definite function on $\mathcal{D}_{+}$which is periodic and OS-positive. Moreover

$$
S(f, g ; s)=\left\langle f, R_{\beta}(s) g\right\rangle
$$


Proof. As was shown in [2], $S(f, g ; s)$ is not only OS-positive, but is also positivedefinite, i.e.

$$
\sum_{k, l=1}^{n} \bar{c}_{k} c_{l} S\left(f_{k}, f_{l} ; s_{k}-s_{l}\right) \geq 0
$$

for all terminating sequences $f_{1}, \ldots, f_{n} \in \mathcal{D}_{+} ; s_{1}, \ldots, s_{n} \in \mathbb{R}$ and $c_{1} \ldots, c_{n} \in \mathbb{C}$. From this condition we obtain that

$$
|S(f, g ; s)|^{2} \leq S(f, f ; 0) S(g, g ; 0) \leq \text { const } \cdot\|f\|^{2}\|g\|^{2}
$$

Since $S(f, g ; s)$ is bilinear and symmetric, there exists a bounded and positive operator $R_{\beta}(s)$ on $\mathcal{D}_{+}$such that

$$
S(f, g ; s)=\left\langle f, R_{\beta}(s) g\right\rangle
$$

Moreover, the function $s \rightarrow R_{\beta}(s)$ is positive-definite, OS-positive and weakly periodic.

Corollary 4. Let $\xi_{s}^{\beta}$ be the Gaussian process indexed by $\mathcal{D}_{+}$defined by

$$
E\left(<\xi_{s}^{\beta}, f>\right)=0, E\left(<\xi_{s_{1}}^{\beta}, f_{1}><\xi_{s_{2}}^{\beta}, f_{2}>\right)=\frac{1}{2}\left\langle f_{1}, R_{\beta}\left(s_{2}-s_{1}\right) f_{2}\right\rangle
$$

Then for $f_{1}, f_{2} \in \mathcal{D}_{+}$

$$
G\left(f_{1}, f_{2} ; s_{2}-s_{1}\right)=E\left(e^{i<\xi_{s_{1}}^{\beta}, f_{1}>} e^{i<\xi_{s_{2}}^{\beta}, f_{2}>}\right)
$$

Similarly, for $f_{1}, \ldots, f_{n} \in \mathcal{D}_{+}$

$$
G\left(f_{1}, \ldots, f_{n} ; s_{1}, \ldots, f_{n}\right)=E\left(e^{i \sum_{k=1}^{n}<\xi_{s_{k}}^{\beta}, f_{k}>}\right) .
$$

Using OS-positivity, and periodicity of $S(f, g ; s), f, g \in \mathcal{D}_{+}$, we can show that [2]

$$
S(f, g ; s)=\int_{0}^{\infty}\left(e^{-s p}+e^{(\beta-s) p}\right) d \nu_{f, g}(p)
$$

where $\nu_{f, g}$ is a finite, real-valued measure on $[0, \infty)$. Now in terms of $\nu_{f, f}$, we can formulate the following continuity property of the process $\xi_{s}^{\beta}$ :

THEOREM 5. If $f \in \mathcal{D}_{+}$is such that $m(f)=\int_{0}^{\infty} p d \nu_{f, f}(p)<\infty$, then the coordinate process $\xi_{s}^{f}:=<\xi_{s}^{\beta}, f>$ has a version (denoted by the same symbol) with Hölder continuous paths. More precisely, for any $0<\gamma<1 / 2$ there exists an integrable random variable $d(f, \gamma)$ such that

with probability one.

$$
\left|\xi_{s}^{f}-\xi_{s^{\prime}}^{f}\right| \leq d(f, \gamma)\left|s-s^{\prime}\right|^{\gamma}
$$

Proof. Since

$$
\begin{aligned}
|S(f, f ; l)-S(f, f ; 0)| & =\left|\int_{0}^{\infty}\left(\left(e^{-l p}-1\right)+\left(e^{-(\beta-l) p}-e^{-\beta p}\right)\right) d \nu_{f, f}(p)\right| \\
& \leq 2|l| \int_{0}^{\infty} p d \nu_{f, f}(p)=2 m(f)|l|
\end{aligned}
$$

it follows that

$$
\left|E\left(e^{z\left|\xi_{s+l}^{f}-\xi_{s}^{f}\right|}\right)\right| \leq E\left(e^{|z|\left(\xi_{s+l}^{f}-\xi_{s}^{f}\right)}\right)+E\left(e^{-|z|\left(\xi_{s+l}^{f}-\xi_{s}^{f}\right)}\right)=2 e^{\frac{|z|^{2}}{2} E\left(\left(\xi_{s+l}^{f}-\xi_{s}^{f}\right)^{2}\right)}
$$




$$
\leq 2 e^{|z||S(f, f ; 0)-S(f, f ; l)|} \leq 2 e^{2|z||l| m(f)} .
$$

On the other hand, by the Cauchy integral formula

$$
\left|\xi_{s+l}^{f}-\xi_{s}^{f}\right|^{n}=\frac{n !}{2 \pi i} \int_{C_{r}} \frac{e^{z\left|\xi_{s+l}^{f}-\xi_{s}^{f}\right|}}{z^{n+1}} d z .
$$

Thus

$$
E\left(\left|\xi_{s+l}^{f}-\xi_{s}^{f}\right|^{n}\right) \leq 2 n ! \frac{e^{2 r^{2}|l| m(f)}}{r^{n}} .
$$

Now, taking $r=|l|^{-1 / 2}$ we obtain $E\left(\left|\xi_{s+l}^{f}-\xi_{s}^{f}\right|^{n}\right) \leq c_{n}|l|^{n / 2}$. The assertion follows by the application of the Kolmogorov continuity test.

Definition 3. A periodic stochastic process $\xi_{s}$ indexed by $\mathcal{D}_{+}$has the two-sided Markov property on the circle $K_{\beta}$ (the circle with radius $\beta$ ) iff for all $r, s \in K_{\beta}$

$$
E_{[s, r]} E_{[r, s]}=E_{\{r, s\}} E_{[r, s]}
$$

where for $I \subset K_{\beta}, E_{I}$ denotes conditional expectation with respect to the $\sigma$-algebra generated by $\left\{\xi_{s}, s \in I\right\}$ and $\{r, s\}=\{s, r\}$ is the set consisting of the two elements $r, s$.

THEOREM 6. $\xi_{s}^{\beta}$ has a version with the two-sided Markov property on the circle $K_{\beta}$.

Proof. The covariance operator $R_{\beta}(s)$ has the form [2]

$$
R_{\beta}(s)=\frac{e^{-s \mathbf{h}}+e^{-(\beta-s) \mathbf{h}}}{1-e^{-\beta \mathbf{h}}}, \quad \mathbf{h} \geq \varepsilon
$$

Let us introduce the new scalar product in $\mathcal{D}_{+}$given by $\langle f, g\rangle_{\beta}:=\left\langle f,\left(1-e^{-\beta \mathbf{h}}\right)^{-1} g\right\rangle$. The norms $\|\cdot\|$ and $\|\left.\cdot\right|_{\beta}$ are obviously equivalent. Let $\tilde{\xi}_{s}$ be the Gaussian process indexed by $\left(\mathcal{D}_{+},\langle\cdot, \cdot\rangle_{\beta}\right)$ with zero mean and covariance operator $\tilde{R}_{\beta}(s)=e^{-s \mathbf{h}}+e^{-(\beta-s) \mathbf{h}} \cdot \tilde{\xi}_{s}$ is stochastically equivalent to $\xi_{s}^{\beta}$ and by the result of [9] it satisfies two-sided Markov property on $K_{\beta}$.

4. KMS structure generated by thermal process. Let $\xi_{s}^{\beta}$ be a Gaussian process constructed above and let $(Q, \Sigma, \mu)$ be its underlying probability space. Since the process is stationary, $u(t)$ defined by

$$
u(t)\left(e^{i<\xi_{s_{1}}^{\beta}, f_{1}>} \cdots e^{i<\xi_{s_{n}}^{\beta}, f_{n}>}\right)=e^{i<\xi_{s_{1}+t}^{\beta}, f_{1}>} \cdots e^{i<\xi_{s_{n}+t}^{\beta}, f_{n}>}
$$

extends to a one parameter group of unitary operators on $L^{2}(Q, \Sigma, \mu)$. By periodicity, $u(\beta)=I$. Since the process is symmetric, $\Theta$ defined by

$$
\Theta\left(e^{i<\xi_{s_{1}}^{\beta}, f_{1}>} \cdots e^{i<\xi_{s_{n}}^{\beta}, f_{n}>}\right)=e^{i<\xi_{-s_{1}}^{\beta}, f_{1}>} \cdots e^{i<\xi_{-s_{n}}^{\beta}, f_{n}>}
$$

extends to a unitary operator on $L^{2}(Q, \Sigma, \mu)$ such that $\Theta^{2}=I$. Finally, since the process is OS-positive

$$
\langle\Theta F, F\rangle_{L^{2}} \geq 0
$$

for all $F \in L^{2}\left(Q, \Sigma_{[0, \beta / 2]}, \mu\right)$ where for $S \subset \mathbb{R}, \Sigma_{S}$ denotes the $\sigma$-algebra generated by $\left\{\xi_{s}^{\beta}: s \in S\right\}$. 
TheOREM 7. Let $\xi_{s}^{\beta}$ be a Gaussian, periodic (with period $\beta$ ), OS-positive stochastic process indexed by $\mathcal{D}_{+}$. Then there exist: a Hilbert space $\mathcal{H}_{\xi}$ with a unit vector $\Omega_{\xi}$, a weakly continuous one-parameter group of unitary operators $U_{\xi}(t)$ and a von Neumann algebra $\mathcal{M}_{\xi}$ of operators acting on $\mathcal{H}_{\xi}$ such that $\Omega_{\xi}$ is cyclic and separating for $\mathcal{M}_{\xi}$ and $\alpha_{t}^{\xi}(M)=U_{\xi}(t) M U_{\xi}(t)^{*}$ is the modular automorphism group associated with $\Omega_{\xi}$.

P r o of. On the space $L^{2}\left(Q, \Sigma_{[0, \beta / 2]}, \mu\right)$ define a sesquilinear form by

$$
\langle F, G\rangle=\langle\Theta F, G\rangle_{L^{2}}
$$

By OS-positivity, it is positive semi-definite. Let

$$
N=\left\{F \in L^{2}\left(Q, \Sigma_{[0, \beta / 2]}, \mu\right):\langle F, F\rangle=0\right\}
$$

Then

$$
D=L^{2}\left(Q, \Sigma_{[0, \beta / 2]}, \mu\right) / N
$$

is a pre-Hilbert space with respect to the inner product

$$
\langle[F],[G]\rangle=\langle F, G\rangle
$$

where $[F]$ denotes the class containing $F$. $\mathcal{H}_{\xi}$ is defined as a Hilbert space completion of $D$ and $\Omega_{\xi}=[1]$. Let $D_{t}$ be the linear space generated by vectors $[F]$ for $F \in$ $L^{2}\left(Q, \Sigma_{[0, \beta / 2-t]}, \mu\right), t \in[0, \beta / 2]$. For every $t \in[0, \beta / 2]$ we can define the linear operator $p(t)$ with domain $D_{t}$ by

$$
p(t)[F]=[u(t) F]
$$

and we can show that $\left(p(t), D_{t}\right)$ form a symmetric local semigroup ([8]). Hence there exists a unique self-adjoint operator $H_{\xi}$ on $\mathcal{H}_{\xi}$ such that $p(t)=e^{-t H_{\xi}} . U_{\xi}(t)$ is defined by $U_{\xi}(t)=e^{i t H_{\xi}}$. Let now $F_{0} \in L^{\infty}\left(Q, \Sigma_{0}, \mu\right)$. Then

$$
\pi_{0}\left(F_{0}\right)[F]=\left[F_{0} F\right]
$$

defines a bounded operator on $\mathcal{H}_{\xi}$ and

$$
\mathcal{M}_{0}=\left\{\pi_{0}\left(F_{0}\right): F_{0} \in L^{\infty}\left(Q, \Sigma_{0}, \mu\right)\right\}
$$

is a von Neumann algebra of operators on $\mathcal{H}_{\xi}$. Let $\mathcal{M}_{\xi}$ be the von Neumann algebra generated by elements

$$
e^{i t_{1} H_{\xi}} B_{1} e^{-i t_{1} H_{\xi}} \ldots e^{i t_{n} H_{\xi}} B_{n} e^{-i t_{n} H_{\xi}}
$$

with $t_{j} \in \mathbb{R}, B_{j} \in \mathcal{M}_{0}$. Then $\Omega_{\xi}$ is cyclic and separating for $\mathcal{M}_{\xi}([7])$. Using the properties of the process $\xi_{s}^{\beta}$ we can now define the modular conjugation and modular group corresponding to $\Omega_{\xi}$. Let

$$
\Theta_{\beta / 4}=u(\beta / 4) \Theta u(-\beta / 4)
$$

and

$$
J_{\xi}[F]=\left[\overline{\Theta_{\beta / 4} F}\right]
$$

Then

$$
\left\langle J_{\xi}[F], J_{\xi}[G]\right\rangle=\left\langle\Theta \overline{\Theta_{\beta / 4} F}, \overline{\Theta_{\beta / 4} G}\right\rangle_{L^{2}}=\langle G, \Theta F\rangle_{L^{2}}=\langle[G],[F]\rangle
$$

since $\Theta_{\beta / 4}$ commutes with $\Theta$ by periodicity. Hence $J_{\xi}$ can be extended to an antiunitary operator on $\mathcal{H}_{\xi}$ such that $J_{\xi}^{2}=I$. Computing the action of $J_{\xi}$ on $\mathcal{M}_{\xi} \Omega_{\xi}, M \in \mathcal{M}_{\xi}$ we 
can show that

$$
J_{\xi} M^{*} \Omega_{\xi}=e^{(-\beta / 2) H_{\xi}} M \Omega_{\xi}
$$

Now since $\mathcal{M}_{\xi} \Omega_{\xi}$ is a core for $e^{(-\beta / 2) H_{\xi}}([7]), J_{\xi}$ defined above is the modular conjugation operator and the corresponding modular operator $\Delta_{\xi}$ can be identified with $e^{(-\beta / 2) H_{\xi}}$.

Let $\left(\mathcal{H}_{\omega}, \pi_{\omega}, \Omega_{\omega}\right)$ be the GNS representation defined by quasi-free KMS state $\omega$. Then $\Omega_{\omega}$ is cyclic and separating for $\pi_{\omega}(W(\mathcal{D}, \sigma))^{\prime \prime}$. We are going to show that in the case of quasi-free state the modular structure constructed from the process $\xi_{s}^{\beta}$ is unitarily equivalent to the canonical modular structure defined by KMS state $\omega$. Thus all relevant information about KMS structure is contained in the (commutative) stochastic process $\xi_{s}^{\beta}$. To obtain this result we need the following property of the dynamic $e^{i t h}$, proved in [2].

TheOREM 8. Let $\mathbf{h}$ be $C$-real on $\mathcal{D}$ and let $e(\{0\})=0$, where de $(\lambda)$ is the spectral measure of $\mathbf{h}$. Then the family

is dense in $\mathcal{D}$.

$$
\left\{\sum_{k=1}^{n} e^{i t_{k} \mathbf{h}} f_{k}: n \in \mathbb{N}, t_{k} \in \mathbb{R}, f_{k} \in \mathcal{D}_{+}\right\}
$$

Now we have:

Theorem 9 ([2]). For quasi-free KMS state $\omega$ defined on the Weyl algebra $W(\mathcal{D}, \sigma)$ the canonical modular structure is unitarily equivalent to the modular structure constructed from the stochastic process $\xi_{s}^{\beta}$.

5. Stochastic positivity for quantum fields on a stationary space-time. Let $(M, g)$ be a stationary space-time, i.e. $(M, g)$ is space nad time orientable with a global time-like Killing vector field $X$. Thus, $(M, g)$ can be always realized as $(\mathbb{R} \times \mathcal{C}, g)$ where $(\mathcal{C}, \widehat{g})$ is a Riemannian 3 -manifold and

$$
g=\left(\begin{array}{cc}
a^{2}-b^{i} b_{i} & -b_{i} \\
-b_{i} & -\widehat{g}_{i j}
\end{array}\right)
$$

with a scalar field $a$ (laps field) and a vector field $b$ (shift field) satisfying

$$
a>0, \quad a^{2}-\widehat{g}(b, b)>0
$$

and with the Killing vector field

$$
X:=\frac{\partial}{\partial t}=a N(\mathcal{C})+b
$$

where $N(\mathcal{C})$ is a unit future-pointing normal vector field to $\mathcal{C}$. If $(M, g)$ is globally hyperbolic, then $\mathcal{C}$ can be chosen to be a global Cauchy surface. On $(M, g)$ we consider the covariant Klein Gordon equation

$$
\left(g^{\mu \nu} \nabla_{\mu} \nabla_{\nu}+m^{2}+V\right) \varphi=0
$$

Given some Cauchy surface $\mathcal{C}$, let

$$
D(\mathcal{C})=C_{0}^{\infty}(\mathcal{C})+C_{0}^{\infty}(\mathcal{C})
$$


be the space of real smooth Cauchy data of compact support. Then, by the Leray's theorem [5], the Cauchy data $\Phi \in D(\mathcal{C})$ given by

$$
\Phi=\left(\begin{array}{l}
f \\
p
\end{array}\right)
$$

define a unique solution $\varphi$ of the Klein Gordon equation having compact support on every other Cauchy surface and such that

$$
\left.\varphi\right|_{\mathcal{C}}=f,\left.\quad N(\mathcal{C}) \varphi\right|_{\mathcal{C}}=p
$$

Thus, we may view time evolution as a one-parameter group

$$
T_{t}: D(\mathcal{C}) \rightarrow D(\mathcal{C})
$$

Moreover, $T_{t}$ preserves the symplectic form

$$
\widehat{\sigma}\left(\Phi_{1}, \Phi_{2}\right)=\int_{\mathcal{C}}\left(f_{1} p_{2}-p_{1} f_{2}\right) d \eta(\mathcal{C})
$$

where $\eta(\mathcal{C})$ is the Riemannian volume element on $(\mathcal{C}, \widehat{g})$ and

with $\widehat{h}=-\mathbf{g A}$ and

$$
\left.\frac{d}{d t} T_{t} \Phi\right|_{t=0}=-\widehat{h} \Phi
$$

$$
\mathbf{A}=\left(\begin{array}{cc}
-\left(\partial^{i} a\right) \partial_{i}+a\left(m^{2}-\Delta(\mathcal{C})+V\right) & -\left(\nabla_{i} b^{i}+b^{i} \partial_{i}\right) \\
b^{i} \partial_{i} & a
\end{array}\right) ; \quad \mathbf{g}=\left(\begin{array}{cc}
0 & 1 \\
-1 & 0
\end{array}\right)
$$

where $\Delta(\mathcal{C})$ is the Laplace-Beltrami operator on $(\mathcal{C}, \widehat{g})$ and $\nabla_{i}$ is the covariant derivative on $(\mathcal{C}, \widehat{g})$. In order to apply previous results, we need a Hilbert space $\left(\mathcal{D},\langle,\rangle_{\mathcal{D}}\right)$ containing $D(\mathcal{C})$, such that $\widehat{\sigma}(\cdot, \cdot)=\operatorname{Im}\langle\cdot, \cdot\rangle_{\mathcal{D}}$ and $T_{t}=e^{i t \mathbf{h}}$, where $\mathbf{h}$ is self-adjoint on $\mathcal{D}$. The result of Kay ([6]) shows that such a one-particle Hilbert space structure exists under the following (mass gap) assumptions:

1. $V$ is stationary and $\inf V(x)+m^{2}>0$,

2. $\inf a>0$ on $\mathcal{C}$,

3. $\inf \left(a-b^{i} b_{i} / a\right)>0$ on $\mathcal{C}$.

The above assumptions imply also that the generator $\mathbf{h}$ has a bounded inverse.

Theorem 10 ([2]). Let $\left(\mathcal{D}, e^{i \mathbf{t h}}\right)$ be the one-particle Hilbert space structure corresponding to the Klein Gordon equation on a globally hyperbolic stationary spacetime $(M, g)$. There exists a complex conjugation $C$ on $\mathcal{D}$ such that $\mathbf{h}$ is $C$-real.

In the case of a static space-time, we can realize $(M, g)$ as above, but with the vector field $b=0$. Then the matrix A becomes diagonal

$$
\mathbf{A}=\left(\begin{array}{cc}
A & 0 \\
0 & a
\end{array}\right)
$$

with

$$
A=-\left(\partial^{i} a\right) \partial_{i}+a\left(m^{2}-\Delta(\mathcal{C})+V\right)
$$

In this case the one-particle structure can be described in a more explicit way. As was shown by Kay $([6]), \mathcal{D}$ can be identified with $L^{2}(\mathcal{C}, d \eta)$ and the space $D(\mathcal{C})$ of Cauchy 
data is mapped into $L^{2}(\mathcal{C}, d \eta)$ by

$$
(f, p) \rightarrow Y f+i Y^{*-1} p
$$

where

$$
Y=\left(\bar{a}^{1 / 2} \bar{A} \bar{a}^{1 / 2}\right)^{-1 / 4} \bar{a}^{1 / 2}
$$

and $\bar{B}$ means closure of operator $B$. The one-particle hamiltonian $\mathbf{h}$ is now given by

$$
\mathbf{h}=\left(\bar{a}^{1 / 2} \bar{A} \bar{a}^{1 / 2}\right)^{1 / 2}
$$

and the complex conjugation $C$ is the natural one in $L^{2}(\mathcal{C}, d \eta)$. Then $\mathcal{D}_{+}$is defined as Hilbert space completion in $L^{2}(\mathcal{C}, d \eta)$ of the linear space generated by vectors

$$
\text { Yf }, \quad f \in C_{0, \mathbb{R}}^{\infty}(\mathcal{C})
$$

and similarly, $\mathcal{D}_{-}$is the completion in $L^{2}(\mathcal{C}, d \eta)$ of linear space generated by

$$
Y^{*-1} p, \quad p \in C_{0, \mathbb{R}}^{\infty}(\mathcal{C}) .
$$

From our general results it follows that there exist thermal process indexed by $\mathcal{D}_{+}$and $\mathcal{D}_{-}$. The former we call field thermal process and denote by $\xi_{t}^{\beta}$ and the latter we call momentum thermal process and denote by $\pi_{t}^{\beta}$. It is enough to consider the covariances of these processes for the indexes of the form $Y f$ or $Y^{*-1} p$ with $f, p \in C_{0}^{\infty}(\mathcal{C})$. Thus we obtain

$$
\begin{aligned}
E\left(<\xi_{t}^{\beta}, f><\xi_{s}^{\beta}, g>\right) & =\int_{\mathcal{C} \times \mathcal{C}}(Y f)(x) R^{\beta}(t, s ; x, y)(Y g)(y) d \eta(x) d \eta(y) \\
E\left(<\pi_{t}^{\beta}, f><\pi_{s}^{\beta}, g>\right) & =\int_{\mathcal{C} \times \mathcal{C}}\left(Y^{*-1} f\right)(x) R^{\beta}(t, s ; x, y)\left(Y^{*-1} g\right)(y) d \eta(x) d \eta(y)
\end{aligned}
$$

where

$$
R^{\beta}(t, s ; x, y)=\frac{e^{-|t-s| \mathbf{h}}+e^{-(\beta-|t-s|) \mathbf{h}}}{1-e^{-\beta \mathbf{h}}}(x, y)
$$

for $|t-s| \leq \beta$. Let $d E_{\mathbf{h}}(\lambda)$ be the spectral resolution of the operator $\mathbf{h}$ in the Hilbert space $L^{2}(\mathcal{C}, d \eta)$. Then

$$
E\left(<\xi_{t}^{\beta}, f><\xi_{0}^{\beta}, g>\right)=\int_{0}^{\infty} \frac{e^{-t \lambda}+e^{-(\beta-t) \lambda}}{\lambda\left(1-e^{-\beta \lambda}\right)} d\left\langle\bar{a}^{1 / 2} f, E_{\mathbf{h}}(\lambda) \bar{a}^{1 / 2} g\right\rangle
$$

Therefore, if $f$ is such that $\bar{a}^{1 / 2} f \in L^{2}(\mathcal{C}, d \eta)$ then

$$
m(f)=\int_{0}^{\infty} \frac{e^{-t \lambda}+e^{-(\beta-t) \lambda}}{1-e^{-\beta \lambda}} d\left\langle\bar{a}^{1 / 2} f, E_{\mathbf{h}}(\lambda) \bar{a}^{1 / 2} f\right\rangle \leq \mathrm{Const}\|\bar{a} f\|_{L^{2}(\mathcal{C}, d \eta)}^{2}
$$

and we can apply Theorem 5 to conclude that for every such $f$ there exists a version of the coordinate process $<\xi_{t}^{\beta}, f>$ with Hölder continuous paths. Similar arguments work also for the momentum process. Thus we obtain

Corollary 11. Let $(M, g)$ be a static, globally hyperbolic space-time with the laps field a. Then

1. For any $f \in C_{0, \mathbb{R}}^{\infty}(\mathcal{C})$ such that $\bar{a}^{1 / 2} f \in L^{2}(\mathcal{C}, d \eta)$ and $\beta \in(0, \infty]$ there exists a version of the coordinate process $\left\langle\xi_{t}^{\beta}, f>\right.$ such that for every $\gamma \in(0,1 / 2)$ there is 
an integrable random variable $d(f, \gamma)$ such that

$$
\left|<\xi_{t}^{\beta}, f>-<\xi_{s}^{\beta}, f>\right| \leq d(f, \gamma)|t-s|^{\gamma}
$$

with probability one.

2. For any $f \in C_{0, \mathbb{R}}^{\infty}(\mathcal{C})$ such that $\bar{a}^{1 / 2} f \in H_{1 / 2}(\mathcal{C}, d \eta)$ where

$H_{1 / 2}(\mathcal{C}, d \eta)=$ metric completion of $C_{0, \mathbb{R}}^{\infty}(\mathcal{C})$ with respect to the (Sobolev-like) norm

$$
\|f\|_{1 / 2}^{2}=\int_{\mathcal{C} \times \mathcal{C}}\left(\mathbf{h}^{1 / 2} f\right)(x)\left(\mathbf{h}^{1 / 2} f\right)(y) d \eta(x) d \eta(y)
$$

the momentum coordinate process $<\pi_{t}^{\beta}, f>$ for $\beta \in(0, \infty]$ has a version with Hölder continuous paths similarly as in (1).

\section{References}

[1] O. Bratteli and D. Robinson, Operator Algebras and Quantum Statistical Mechanics,Vol. 2, Springer-Verlag, New York, 1981.

[2] R. Gielerak, L. Jakóbczyk and R. Olkiewicz, Stochastically positive structures on Weyl algebras. The case of quasi-free states, BiBoS preprint, Nr 775/5/97.

[3] R. Gielerak and R. Olkiewicz, Gentle perturbations of the free Bose gas. I, J. Stat. Phys. 80 (1995), 875-918.

[4] R. Gielerak and R. Olkiewicz, Gentle perturbations of the free Bose gas. II. The critical regime, J. Math. Phys. 37 (1996), 1268-1286.

[5] J. Leray, Hyperbolic Differential Equations, The Institute for Advanced Studies, Princeton, 1953.

[6] B. S. Kay, Linear spin-zero quantum fields in external gravitational and scalar fields. I., Commun. Math. Phys. 62 (1978), 55-70.

[7] A. Klein and L. J. Landau, Stochastic processes associated with KMS states, J. Funct. Anal. 42 (1981), 368-428.

[8] A. Klein and L. J. Landau, Construction of a unique self-adjoint generator for a symmetric local semigroup, J. Funct. Anal. 44 (1981), 121-137.

[9] A. Klein and L. J. Landau, Periodic Gaussian Osterwalder-Schrader positive processes and the two-sided Markov property on the circle, Pacific J. Math. 94 (1981), 341-367.

[10] J. Manuceau, M. Sirugue, D. Testard and A. Verbeure, The smallest $C^{*}$-algebra for canonical commutation relations, Commun. Math. Phys. 32 (1973), 231-242. 